\title{
Fenómenos y creencias en Aristóteles. Una interpretación sobre el rol metodológico de las éndoxa en la ciencia natural
}

\section{Phenomena and Beliefs in Aristotle. An interpretation on the methodological role of endoxa in natural science}

\author{
Fabián Mié \\ (Consejo Nacional de Investigaciones Científicas y Técnicas. Universidad Nacional del Litoral)
}

Recibido: 10/01/2012

Aceptado: 07/12/2012

\section{Resumen}

Me propongo ofrecer una interpretación unificada de la metodología aristotélica y mostrar que ella se integra en un empirismo híbrido en el cual se combina la doble naturaleza de los phainomena, hechos observacionales y creencias comunes. Polemizaré con la lectura coherentista de la prueba dialéctica de consistencia que se expresa en EN VII 1, y trataré de aclarar qué uso hace Aristóteles de aquella doble fuente de sus investigaciones en algunos textos de ciencia natural ( $P h$. IV 4).

Palabras clave: Aristóteles, metodología, principios, física, dialéctica, fenómenos, creencias, empirismo, coherentismo, prueba

\section{Abstract}

In this paper I aim to offer a unified interpretation of Aristotle's methodology understood as a procedure which is part of his hybrid empiricism in which the double face of phainomena - observed facts and common opinions - is an integral part. I argue against the coherentist lecture on the dialectic proof of consistence (EN VII 1) and explain why is the double face of phaenomena of real use in natural science (Ph. IV 4).

Keywords: Aristotle, methodology, principles, physics, dialectic, phenomena, beliefs, empiricism, coherentism, proof. 
[...] es evidente, pues, que también en la ciencia de la naturaleza hay que intentar delimitar, primeramente, lo concerniente a los principios. Ahora bien, naturalmente, el procedimiento es a partir de las cosas más conocidas y claras para nosotros hacia las más claras y conocidas por naturaleza ( $\left.P h . \mathrm{I} 1,184 \mathrm{a} 14-18^{1}\right)$.

La causa de que seamos pocas veces capaces de ver las cosas sobre las cuales existe acuerdo es la falta de experiencia. Por eso, quienes más han habitado entre las cosas naturales, son más capaces de suponer principios tales que, en la mayoría de los casos, los capacitan para establecer vínculos. Mientras que otros, partiendo de muchos razonamientos, no habiendo observado los hechos reales y dirigiendo la mirada hacia pocos, se manifiestan con ligereza. Se puede ver también a partir de esto cuánto se diferencian los que investigan físicamente de los que lo hacen lógicamente ( $G C$ I 2, 316a5-11).

\section{La dualidad de los phainómena aristotélicos y el problema del método}

Uno de los tópicos de la discusión actual sobre la metodología de Aristóteles atañe al eventual aporte que las creencias comunes o plausibles (éndoxa) están en condiciones de ofrecer a las ciencias particulares. Esta controversia es parte de otra de mayor escala que concierne a si Aristóteles, en su concepción y práctica de la ciencia natural, aplica un método empírico -aunque lo haga de manera deficiente en comparación con los estándares de la epistemología fundacionista que comúnmente se le atribuye- $\mathrm{u}$ otro dialéctico con componentes conceptuales a priori y una justificación meramente coherentista. En el presente artículo me propongo abordar esta cuestión desde la óptica parcial del lugar que ocupan las creencias comunes en la metodología científica de Aristóteles dentro de la investigación de los principios en la ciencia natural.

Uno de los principales autores que se oponen a la lectura coherentista-dialéctica de la metodología de Aristóteles en ciencia natural es Robert Bolton ${ }^{2}$. Él critica los dos aspectos antes señalados: (a) impugna la interpretación de las éndoxa en términos de creencias que contendrían elementos apriorísticos de nuestra compren-

\footnotetext{
1 Soy responsable por las traducciones incluidas en este texto. Cito a Aristóteles según las ediciones de Oxford Classical Texts (OCT); y para las obras adopto las abreviaturas del Greek-English Lexicon (LSJ).

2 Bolton, R.: "The Epistemological Basis of Aristotelian Dialectic", en M. Sim (ed.), From Puzzles to Principles? Essays on Aristotle's Dialectic, Lanham, Lexington, 1999, pp. 57-105, p. 89 (contra Owen, G.E.L.: "Tithénai tà phainómena", en S. Mansion (ed.), Aristote et les Problèmes de Méthode, Paris/Louvain, Éditions de l'Institut Supérieur de Philosophie, 19802, pp. 83-103); Bolton, R.: "Aristotle's Method in Natural Science: Physics I", en L. Judson (ed.), Aristotle's Physics. A Collection of Essays, Oxford, Oxford University Press, 1991, pp. 1-29; Bolton, R.: "Two Standards for Inquiry in Aristotle's De Caelo", en C.A. Bowen and C. Wildberg (eds.), New Perspectives on Aristotle's De Caelo, Leiden/Boston, Brill, 2009, pp. 51-82.
} 
sión; y también (b) rechaza que la justificación del conocimiento demostrativo se alcanzaría al probar la coherencia entre los resultados científicos y cierto núcleo duro de creencias comunes. Creo que ambas críticas a la lectura coherentista son acertadas, y que es lícito (i) rechazar esa lectura en virtud de que, por un lado, compromete a Aristóteles con un conjunto de tesis metodológicas y epistemológicas que no corresponden realmente a lo que es el uso y aporte efectivos de la dialéctica en la ciencia. Pero, además, desde un punto de vista sistemático, (ii) la lectura impugnada le adjudica a Aristóteles un concepto y uso de las éndoxa que las sustrae de un adecuado control racional, en la medida en que las extirpa de la raíz empírica del conocimiento que, en general, es adjudicable a la posición epistemológica del estagirita. En efecto, con la lente del coherentismo no se puede enfocar la dinámica de formación y corrección de las creencias dentro de una imagen empirista del conocimiento, ni resulta posible explicar de qué manera se integran las creencias plausibles tanto en la investigación de los principios como en el control de los resultados de la demostración científica, algo que el estagirita propone en textos programáticos que aquí abordaré. En vista de este estado de la cuestión, me propongo ofrecer una interpretación del método empírico de Aristóteles en ciencia natural que esté en condiciones de dar cuenta de cuál es efectivamente la contribución que prestan las creencias plausibles o comunes, e igualmente pueda explicar cuál es el uso que de ellas se hace en la aplicación científica de la dialéctica prevista en Top. I 2, 101a34b43, restringiéndome en este caso a la ciencia natural. Mi objetivo se verá cumplido si logro mostrar que Aristóteles les reserva a las éndoxa un papel determinado e insustituible en la elaboración del conocimiento demostrativo, sin que ese papel desnaturalice las pretensiones explicativas de la ciencia ni contradiga la tesis empirista que mantiene el estagirita. En orden a ello, analizaré algunos pasajes del corpus que son representativos tanto del restringido predominio del examen dialéctico en algunos contextos como también de los objetivos explicativos que persiguen las teorías científicas en la concepción aristotélica. Trataré de mostrar que entre las investigaciones físicas y las éticas existe una compatibilidad metodológica; y para explicarla -sin, empero, confundir los objetivos del procedimiento analítico de explicación causal y los del procedimiento dialéctico que busca mantener la consistencia entre las creencias- distinguiré la naturaleza doble de los phainómena aristotélicos. Trataré de mostrar, en este sentido, cuál es la incidencia diferenciada que le corresponde a esa doble faz de los fenómenos -cuyo dominio está ocupado por hechos observacionales y creencias comunes- en la elaboración de la ciencia natural. Mi objetivo principal será, entonces, explicar de qué manera ambos aspectos de los fenómenos aristotélicos constituyen complementariamente la metodología científica utilizada en los distintos tratados. En virtud de la mencionada doble compo-

3 Traduzco y comento el pasaje aquí mismo más adelante en el apartado 3. 
sición de los fenómenos, definiré la metodología aristotélica como la que corresponde a un empirismo híbrido, y sostendré que la tesis epistemológica de Aristóteles no admite que la dialéctica cumpla con el papel justificatorio que el estagirita asigna a la construcción de demostraciones, sólo a través de las cuales se prueba que los principios postulados tienen real fuerza explicativa.

\section{La prueba dialéctica de consistencia y su interpretación coherentista}

En este apartado me propongo discutir la lectura coherentista de la dialéctica aristotélica; comenzaré explicitando a rasgos generales las pretensiones de esa lectura. G.E.L. Owen aseveró que cuando Aristóteles aborda la acción humana utilizaría una clase especial de phainómena -las éndoxa- que no coinciden con observaciones ni son hechos puros. El rol que estos últimos factores desempeñarían en la ciencia natural lo jugarían, en las investigaciones éticas, las "creencias" que versan sobre problemas que se inscriben en un orden que exige un procedimiento dialéctico. Owen sostiene que cuando los hechos meramente confirman una teoría a título de ejemplos, ellos ya están interpretados, cargados de teoría; por ende, el recurso a tales "hechos" no podría interpretarse en conformidad con el parámetro de la investigación empírica. De esto sería un signo que los fenómenos utilizados en la teoría sobre la incontinencia no coinciden con los "fenómenos observacionales" ( $A P r$. I 30, 46a20) reclamados por Aristóteles en $A P r$. I 30, 46a17-27 a título de la base de experiencia (empeiría) de la cual es preciso servirse para descubrir los principios de la astronomía ${ }^{4}$.

¿Qué puede justificar a Aristóteles a incluir creencias comunes como los puntos de partida de la investigación de una ciencia particular? Owen responde a esto restringiendo la legitimidad del uso de tales creencias a solo aquellas investigaciones en las cuales hay que tomar en cuenta opiniones comunes o afirmaciones corrientes, lo que delinearía su carácter dialéctico (logikôs), por oposición al empírico y más específico (physikôs) que adquirirán, por ejemplo, las investigaciones destinadas a encontrar los principios de la astronomía, la meteorología o la física. La lectura coherentista que Owen hace de la incidencia metódica que tendría la dialéctica sobre la ciencia aristotélica, y la derivada distinción entre dos métodos, dependen de la contraposición que él traza entre hechos observacionales y creencias plausibles. Creo que este punto amerita una discusión a fin de evaluar las supuestas dos

\footnotetext{
4 Owen, G.E.L.: "Tithénai tà phainómena", cit., espec. pp. 102 s., 84 s., y notas. La interpretación de Augustin Mansion sobre el método "conceptual" de la física aristotélica influyó decisivamente en Owen. Mansion, A.: Introduction à la physique aristotélicienne, Louvain-La-Neuve, Éditions de l'Institut Supérieur de Philosophie, 19872, p. 211: «Tout s'y réduit en général à des analyses plus ou moins poussées de concepts $[\ldots]$ ».
} 
metodologías de Aristóteles. Quisiera mostrar aquí que aquella restricción impuesta por Owen para justificar el uso de las creencias y la aplicación científica de la dialéctica no corresponde a la práctica de Aristóteles ni a su teoría, en la medida en que el estagirita asigna una contribución epistemológica a las creencias comunes, ubicándolas en la base inductiva de la investigación científica, más precisamente, en la instancia pre-demostrativa de una ciencia particular ${ }^{5}$. Esto nos debe conducir a trazar de un modo más preciso la oposición entre logikôs vs physikôs, la cual no puede entenderse como una oposición entre dialéctica vs física-como lo hizo Terence Irwin-, dada la vasta aplicación de la dialéctica en la ciencia natural. Propondré entender la primera oposición como una entre un procedimiento propiamente científico - "físico"-, adecuado al carácter empirista híbrido que le adjudicaré a la base inductiva a partir de la cual Aristóteles busca descubrir los principios de las ciencias, y otro procedimiento de carácter conceptual -"lógico"-, que no practica una descripción satisfactoria y completa de los hechos relevantes, partiendo frecuentemente de supuestos dogmáticos tomados de un dominio heterogéneo6.

Owen y la crítica posterior individualizaron dos pasajes centrales del corpus para discutir las dos metodologías que se le adjudican a Aristóteles. A fin de poder tenerlos en cuenta, a continuación traduciré y en el curso de mi labor posterior trataré de aclarar esos dos documentos metodológicos principales, o sea, el ya aludido de $A P r$. I 30 y su homólogo de EN VII 1, 1145b2-6, que expresaría el método dialéctico. El pasaje estandarte de la metodología empírica reza:

En cambio, para cada ciencia son propios la mayoría $<$ de los principios $>$. Por eso, la experiencia relativa a cada cosa es la que proporciona los principios; aludo, por ejemplo, a que la experiencia astronómica es de la ciencia astronómica (en efecto, una vez que se aprehendieron suficientemente los fenómenos, así se encontraron las demostraciones astronómicas). E igualmente sucede con cualquier otro arte y ciencia. De manera que, una vez que se hayan aprehendido las cosas que se dan (tà hypárchonta) en cada cosa, es ya asunto nuestro poner de manifiesto fácilmente las demostraciones. Pues si en la indagación de lo que realmente se da en las cosas ${ }^{7}$ no se dejó nada de lado, seremos capaces, acerca de todo aquello de lo cual hay demostración, de encontrarla, y

\footnotetext{
5 Para la instancia pre-demostrativa, véase Ferejohn, M.: The Origins of Aristotelian Science, New Haven/London. Yale University Press, 1991, pp. 19 ss., 38-61.

6 Cleary, J. J.: Aristotle and Mathematics. Aporetic Method in Cosmology and Metaphysics, Leiden, Brill, 1995, p. 136, n. 112; Charlton, W.: Aristotle, Physics. Books I and II. Translated with Introduction, Commentary, Note on Recent Work, and Revised Bibliography, Oxford, Oxford University Press, 1992, pp. x-xii.

7 La expresión tôn alethôs hyparchónton toîs prágmasin indica las propiedades por sí de un objeto, que según $A P o$. I 6, 74b6-7; I 10, $76 \mathrm{~b} 4$ son necesarias y aquello cuya pertenencia a un sujeto es objeto de la demostración. Debo esta aclaración a Eduardo Mombello, así como muchas otras valiosas sugerencias y correcciones relativas a las traducciones incluidas en este texto, las cuales, naturalmente, no lo comprometen a él.
} 
demostrar; mientras que acerca de aquello de lo cual por naturaleza no hay demostración, de ponerlo de manifiesto ${ }^{8}$ ( $A P r$. I 30, 46a17-27) ${ }^{9}$.

El documento que correspondería al método dialéctico mantiene lo siguiente:

Pero como en los demás casos es necesario, si se establecen los fenómenos (tithéntas tà phainómena) y primero se examinaron las aporías, probar (deiknýnai) de esta manera, sobre todo, todas las opiniones plausibles (pánta tà éndoxa) acerca de estas pasiones; pero si no, la mayoría y las más importantes; pues siempre que se hayan resuelto las dificultades y hayan quedado firmes las opiniones plausibles, se habría probado suficientemente (dedeigménon àn eíe hikanôs) (EN VII 1, 1145b2-7)10.

Antes de considerar más pormenorizadamente este segundo pasaje, puede ser conveniente trazar algunos lineamientos generales sobre la dialéctica. En Top., la dialéctica es una técnica argumentativa que, para efectuar su examen de las tesis propuestas por el interlocutor, apela a opiniones comunes que no pertenecen a un área particular del conocimiento (Top. I 1, 100a18-20) y las usa como puntos de partida y criterios de admisibilidad de las opiniones examinadas 11 . De tal manera, el silogismo dialéctico (100a29-b23) apunta a someter a examen las creencias en términos de una prueba de la consistencia que cierta proposición mantiene con un cuerpo mayor de creencias bien acreditadas, en relación con el cual, como condición necesaria para que pueda admitirse aquella proposición, la creencia que ésta expresa no debe hallarse en contradicción. Cuando la dialéctica se aplica a las aporías que se plantean en un determinado campo del conocimiento no resigna su carácter inespecífico que distingue sus silogismos respecto de los demostrativos (100a27-30). El objetivo de esa aplicación se mantiene dentro de los lineamientos del razonamiento dialéctico, pues se tratará de dejar en pie las proposiciones que mantienen la mayor consistencia con un cuerpo de creencias firmemente establecido. Tal es la manera en que se buscar resolver la dificultad mediante el procedimiento dialéctico, es decir, rechazando las creencias que carecen de la consistencia necesaria. Esto no excluye que, tras el examen dialéctico, una opinión que prima facie parecía contradecir aserciones acreditadas resulte finalmente aceptada ${ }^{12}$. La regla

\footnotetext{
8 Se trata del conocimiento no demostrativo de los principios de una demostración (APo. I 3, 72b19 ss.; II 19, $100 \mathrm{~b} 8$ ss.).

9 PA I 1, 639b6-11; I 5, 645b1-3; HA I 6, 491a10-14.

$10 \mathrm{Ph}$. IV 4, 211a7-11; EE I 6, 1216b26-35 (ambos traducidos y comentados aquí mismo más adelante).

11 Top. VIII 5, 159b8-9: «Puesto que quien razona correctamente prueba lo sometido a discusión a partir opiniones más plausibles y mejor conocidas». También 159b13-14.

12 Éste puede ser el caso en el cual, cuando no contamos con una opinión acerca de cierto tema (Top. I 11, 104b30-31), un experto plantea un problema que, empero, no constituye una opinión contraria a un éndoxon, no es un juicio paradójico (hypólepsis parádoxos, 104b19, b34) perteneciente a un área
} 
general de la construcción de razonamientos correctos para quien busca ejercitarse y ensayar no es otra que la examinación de la opinión propuesta -sea ésta plausible o no- en términos de la consistencia que ella mantenga con las opiniones más plausibles a que recurre el dialéctico en su rol de interrogador (VIII 5). La admisión posterior de una opinión problemática, eventualmente proveniente de un campo especializado del conocimiento, puede acabar justificando, entonces, porque su eventual rechazo se constata que conduciría a una contradicción de mayor peso con cierto núcleo de opiniones comunes ampliamente admitidas.

Aristóteles advierte prontamente, para diferenciar el razonamiento genuinamente dialéctico respecto del erístico, que no todo éndoxon aparente es un éndoxon real (Top. I 1, 100b26); y sólo este último puede constituirse en el genuino parámetro de evaluación para las opiniones sometidas a examen. Lo que es realmente una opinión plausible puede entenderse en vinculación con lo que Aristóteles llama "una opinión más/máximamente plausible" (e.g. Top. VIII 5, 159b8-9, b13-14; SE 33, 182b38), cuya inclusión en las premisas de un razonamiento dialéctico forma parte, simplemente, de la elaboración correcta de un silogismo de este tipo (APo. I 19, 81b18-20). Así, la individuación de un núcleo de creencias comunes fuertemente establecido es una parte esencial del examen dialéctico estándar.

Una suposición central de Aristóteles es que a partir de la aceptación mayoritaria de una creencia parece poder inferirse su verdad. Aristóteles lo expresa en varias ocasiones 13 :

Pues las cosas que parecen a todos, afirmamos que son así (EN X 2, 1172b36-1173a1).

En los lineamientos básicos de la dialéctica que he trazado resalta ya una diferencia entre esa técnica de argumentación y la labor científica. Pues en la ciencia no se utiliza como criterio de justificación primero, único o dominante, la consistencia; e incluso ésta puede ser completamente irrelevante para la justificación de una teoría científica, al menos si tenemos en cuenta los objetivos que persigue quien construye argumentos dialécticos en vista del ensayo y la investigación, tal como Aristóteles los especifica en Top. VIII 514. En efecto, aparte de otros parámetros de evaluación de teorías - como la simplicidad o la consistencia con otras teorías ya

del conocimiento especializado, es decir, no alcanza a ser lo que Aristóteles llama una "tesis" en sentido estricto, que juzga necio admitir en cuanto que ella es contraria a la opinión de la mayoría (104b22-24). Tal es, en efecto, uno de los casos posibles en que puede plantearse un próblema (104b4; la enumeración completa de los casos, en 104b1-17). En tal situación, no contaríamos con una opinión acreditada con la cual contrastar la afirmación del experto.

13 Creo que éste es el motivo de fondo por el cual Aristóteles juzga necio admitir las opiniones paradójicas.

14 Irwin, T. H.: Aristotle's First Principles, Oxford, Oxford University Press, 1988, p. 39, contrapone dialéctica y ciencia, desde ese punto de vista. 
establecidas-, Aristóteles reconoce que la fuerza explicativa que le concedemos a cierto principio de una teoría científica no depende primariamente de la relación de consistencia que él mantenga con nuestras creencias; ya que la justificación científica depende de que por medio de ciertos principios se alcancen a explicar los hechos. Sin comprometerme ahora con una cierta lectura realista de estas tesis de Aristóteles ${ }^{15}$, creo que podemos admitir que algunas ideas del estagirita acerca de la independencia que le corresponde a la realidad con respecto a nuestras creencias, así como su afirmación de la asimetría que también existe entre el hecho que hace verdadero a un enunciado y la verdad de nuestras creencias, desembocan en un marcado contraste entre los criterios de aceptación de tesis válidos en la dialéctica y en la ciencia. En todos los criterios que Irwin 16 individúa como aceptados por Aristóteles para considerar justificada una teoría científica, el peso recae exclusivamente sobre las "apariencias observacionales relevantes", lo que no corresponde a patrón de consistencia dialéctica alguno. Pienso que, incluso guardando serios reparos ante la manera en que Irwin traza una oposición metodológica entre el procedimiento coherentista de análisis de conceptos y el procedimiento empírico de explicación causal, hay que mantener algunos elementos de ese contraste entre la justificación dialéctica y la justificación científica de una teoría17.

Como ya señalé, Bolton 18 buscó una inteligente solución alternativa. Él piensa que la dialéctica o, más precisamente, lo que él llama "peirástica", resulta necesaria porque las creencias comunes están en conformidad con los datos observacionales obtenidos hasta un cierto momento, pero sería insuficiente por esa misma razón. Ésta es una interpretación parcialmente aceptable; sin embargo, a mi juicio, tiene dos problemas: (1) aproxima demasiado la dialéctica peirástica a la ciencia, en cuanto a la manera empirista de obtener la justificación de una creencia o de una teoría científica; y (2) no refleja el rol conceptual que poseen las creencias comunes, y que me parece es el que torna a la dialéctica un componente necesario de las ciencias, tanto para el descubrimiento de sus principios como para la justificación de sus resultados. Una porción de mi trabajo consistirá, por lo tanto, en formular una interpretación sobre el uso científico de la dialéctica donde se preserve la antes mencionada contraposición entre una justificación mediante la consistencia y otra mediante una demostración estricta, sin empero adjudicar a Aristóteles una dualidad metodológica a la que no me siento inclinado por las razones que aduciré acerca de la naturaleza y el uso de las éndoxa en las ciencias particulares.

\footnotetext{
15 Como lo hace Irwin, T. H.: Aristotle's First Principles, cit., pp. 30 ss., 117 ss., 127 ss., al hablar de un "realismo metafísico" correspondiente a un "empirismo ingenuo" en Aristóteles.

16 Irwin, T. H.: Aristotle's First Principles, cit., pp. 33 ss. (con los pasajes).

17 Me veo dispensado aquí de intentar confirmar en detalle los aspectos generales de la teoría de la ciencia que le he adjudicado al estagirita.

18 Bolton, R.: "The Epistemological Basis of Aristotelian Dialectic", cit., pp. 95-99.
} 
Retornemos ahora a uno de los principales documentos que pretendo discutir: EN VII 1. Enrico Berti19 sostuvo que allí el examen de las éndoxa consiste en examinar cierta opinión acreditada a partir de sus consecuencias y de la consistencia que tales consecuencias mantienen con otras éndoxa genuinas; ello permitiría confirmar las opiniones acreditadas que gozan de una mayor aceptabilidad, y, concomitantemente, rechazar las opiniones que son sólo aparentemente "endóxicas", es decir, aquellas aseveraciones que, incluso pudiendo ser verdaderas, no son creídas efectivamente por un grupo relevante de sujetos y, por ello, no son dignas de atención en el marco de una consideración dialéctica. La "demostración suficiente", que resulta de "resolver las dificultades y dejar subsistir las éndoxa" (EN VII 1, 1145b67), no consistiría, para Berti, más que en un examen de coherencia ${ }^{20}$.

El punto problemático de esta interpretación me parece que reside en que, si bien capta lo peculiar del examen dialéctico y encuentra en la prueba de consistencia la fuerza probatoria de un argumento de ese tipo, creo que no nos alcanza para resolver el problema que antes señalé, es decir, el del valor epistemológico de las éndoxa y la justificación del uso científico de la dialéctica. Tal era el problema denunciado por Irwin: que Aristóteles meramente "supondría" que algunas opiniones están autorizadas a desempeñar un rol en la ciencia, en cuanto que ellas captarían la realidad. Es cierto que Aristóteles señala, en algunas oportunidades, que los hombres están equipados naturalmente para captar en la mayor parte de los casos la verdad o para obtener una aproximación correcta a lo que es verdadero (e.g. $R h$. I 1, 1355a10-18; Metaph. II 1, 993a30-b7; EN I 8, 1098b27-29; EE I 6, 1216b30-32).

Berti desarrolla una estrategia para apoyar desde la misma dialéctica esa intuición de Aristóteles, y es la siguiente: Aristóteles intentaría mostrar que (i) por su carácter de ser "lo que se cree mayormente" (Top. I 2, 100b21-23), las éndoxa se cuentan como "opiniones verdaderas en la mayor parte de los casos"; para ello, sostendría que (ii) en virtud de que lo "probable" (eikós) -"lo que es el caso la mayoría de las veces" ( $R h$. II 24, 1402a8-17)- constituye una característica de ciertas creencias que se introducen en las premisas de un entimema, existe un vínculo entre la opinión mayoritariamente aceptada y la verosimilitud, merced a la frecuencia (II 25, 1402b14-16, b20-23); a ello se añade que (iii) lo "verdadero" y lo "verosímil" son captados por una misma facultad de conocimiento (I 1, 1355a14-17), lo que sugiere suplementariamente que existe una vinculación entre "lo que es mayoritariamen-

19 Berti, E.: 'L' uso 'scientifico' della dialettica in Aristotele", reed. en Nuovi studi aristotelici. IEpistemologia, logica e dialettica, Brescia, 2004, Morcelliana, pp. 265-282.

20 A diferencia de Bolton, Berti sostiene que la plausibilidad de todo éndoxon impide que haya un conflicto entre opiniones de ese tipo. Berti, E.: "Il valore epistemologico degli éndoxa secondo Aristotele", reed. en Nuovi studi aristotelici. I- Epistemologia, logica e dialettica, Brescia, 2004, Morcelliana, pp. 317-332. Sin embargo, la repetida caracterización estándar de las éndoxa destaca entre ellas una gradación que va desde aquellas aceptadas por todos, hasta el extremo de otras admitidas sólo por los más notables de entre los expertos (e.g. Top. I 1, 100b21-23; 14, 105a34-b1). 
te aceptado" (éndoxon) y "lo que es casi siempre verdadero"; (iv) todo esto justificaría el "valor epistemológico" de las éndoxa y su utilización particularmente en disciplinas en las cuales los hechos son frecuentes (física, $P h$. II 8, 198b35-36, y ética, EN I 3, 1094b21-22)21.

Si éste fuera el final de la historia, la dialéctica se justificaría plenamente en su uso científico por la aproximación natural humana a la verdad. Sin embargo, como advirtió Bolton, este optimismo aristotélico no impide (a) que ciertos datos perceptivos nuevos, que no son éndoxa, tengan un estatuto epistémico superior al de tales creencias; ni que -añadiría yo- (b) aquella aproximación natural a la verdad sea evaluada por Aristóteles como por sí sola insuficiente en orden a alcanzar el nivel epistémico que se obtiene exclusivamente mediante la demostración de una teoría científica.

Pienso que alguien -como es mi caso- que está convencido de que, sin embargo, las éndoxa son una parte necesaria de la elaboración científica, está obligado a acomodar el conjunto de datos e interpretaciones dispares que he repasado hasta aquí; más aun si se pretende mantener una lectura compatibilista de la metodología aristotélica y se desea preservar para la dialéctica una genuina utilidad científica que, empero, no sobrepase los límites de lo que ese artificio argumentativo puede aportar como mejora a aquella capacidad natural humana de aproximarse a la verdad. La legitimación del uso científico de la dialéctica difícilmente pueda provenir de lo que es la operación argumentativa primariamente examinadora (exetastiké) y probatoria (peirastiké), es decir, "refutativa", dirigida a las opiniones que alegan un saber sin encarnarlo realmente (e.g. $S E$ 34, 183a39). A mi juicio, la principal dificultad para justificar el recurso a las opiniones comunes cuando se elabora una demostración reside en la identificación de los títulos eventuales que podría alegar a su favor la dialéctica en orden a legitimar dicho recurso a las creencias meramente comunes y, además, no necesariamente verdaderas en un contexto donde los silogismos se construyen a partir de principios propios (APo. I 9). Es innegable, pues, que ninguna creencia, incluso una que goce de aceptación completa (consensus omnium), ofrece por sí misma una estructura causal y necesaria de un hecho, lo que es un requisito indispensable para que una proposición pueda desempeñarse como un principio propio aristotélico. En lo siguiente será mi tarea tratar de resolver este problema.

\section{El problema del uso científico de las éndoxa y la "demostración suficiente"}

La "examinación" constituye un procedimiento peculiar de la dialéctica, como lo testimonia el pasaje referido a su uso científico de Top. I 2:

21 Berti, E.: "Il valore epistemologico degli éndoxa secondo Aristotele", cit., pp. 321 ss., 329 s. 
Y además $<$ el tratado es útil $>$ para las cosas primeras 22 de cada ciencia. Pues a partir de los principios apropiados según cada ciencia propuesta es imposible decir algo acerca de ellos, puesto que los principios ${ }^{23}$ son los primeros de todas las cosas; sin embargo, a través de las opiniones plausibles 24 acerca de cada uno es necesario revisar detalladamente lo que a ellos concierne. Y esto es peculiar de, o principalmente apropiado para la dialéctica. En efecto, en virtud de que está diseñada para examinar con detenimiento (exetastiké), guía en el camino hacia los principios de todos los métodos 25 (Top. I 2, 101a36-b4).

El contraste entre el procedimiento de la ciencia y el de la dialéctica que se lee aquí resalta también donde (Top. I 14, 105b30-32) Aristóteles busca aclarar sus respectivos intereses. La argumentación dialéctica está motivada por la consideración de opiniones (pròs dóxan) concurrentes acerca de un tema opinable sobre el cual existen creencias opuestas y resolubles. La ciencia, en cambio, encuentra su motivación en la investigación de la verdad (kat'alétheian) acerca de un tema específico sobre el cual la existencia de opiniones, sean éstas dispares o incluso contradictorias, es, por principio, expresión de una deficiencia en el nivel del conocimiento que se ha alcanzado acerca de la materia en cuestión.

Para resolver el problema que he identificado en el apartado anterior me parece crucial esclarecer el aporte que puede hacer la demostración suficiente, que resulta de resolver las dificultades y dejar subsistir las éndoxa (EN VII 1, 1145b6-7)26.

\footnotetext{
22 Para la referencia de tà prôta, véase Top. I 1, 100a27, a28, b1.

23 Para la referencia de archai a los principios de las ciencias particulares, véase Top. I 1, 100b20; y para la referencia a las ciencias particulares, 101a6 (a7).

24 Para la referencia de éndoxa a la clase de premisas de un razonamiento dialéctico, véase Top. I 1, 100a30; y para su cualificación como creencias plausibles, 100b21-23.

25 Con méthodos, Aristóteles alude al carácter metódico de las ciencias particulares y de otras disciplinas, es decir, a la manera en que cada una de ellas procede, y no tanto al cuerpo de proposiciones que componen una disciplina científica particular (veáse Top. I 3). Pero la dialéctica tiene también un carácter metódico análogo a ellas (I 3, 101b5), y el mismo consiste, tratándose del uso científico de la dialéctica, en proveer un procedimiento (hódos, I 2, 101b4) para conocer los principios de las ciencias particulares. Para "méthodos", I 3, 101b5; I 4, 101b11; I 2, 101a29; EE I 6, 1216b35. Top. I 3 y I 4 comienzan a abordar, por consiguiente, propiamente la cuestión del método dialéctico. Véase Brunschwig, J.: Aristote, Topiques (Livres I-IV). Texte établi et traduit, Paris, Les Belles Lettres, 1967, pp. 116 s.; Zadro, A.: Aristotele, I Topici. Traduzione, introduzione e commento, Napoli, Loffredo, 1974, pp. 315 s.

26 Smith, R.: "Dialectic and Method in Aristotle", en M. Sim (ed.), From Puzzles to Principles? Essays on Aristotle's Dialectic, Lanham, Lexington, 1999, pp. 39-55, p. 41, enfatizó que no puede entenderse que este último texto establezca una prueba mínima, ya que fijaría una condición de máxima (kállista deiknýoito). Para "el método de las éndoxa" en la ética, Barnes, J.: "Aristotle and the Method of Ethics", Revue International de Philosophie, 34, 1980, pp. 490-511, p. 495; una posición similar defendió para la ciencia natural Wieland, W.: Die aristotelische Physik. Untersuchungen über die Grundlegung der Naturwissenschaft und die sprachlichen Bedingungen der Prinzipienforschung bei Aristoteles, Göttingen, Vandenhoeck \& Ruprecht, 19923, pp. 261 ss., passim.
} 
Aristóteles insiste en ese mismo reclamo en Ph. IV 4 (211a7-11), donde advierte que, como resultado del tipo de prueba o demostración que él tiene a la vista $-\mathrm{y}$ pienso que en ambos textos él apunta a la misma clase de demostración de una teoría por recurso a la consideración de su consistencia con las creencias aceptadas-, las éndoxa resultarán establecidas o confirmadas como verdaderas (tà dokoûnta hypárchein tôi tópoi hypárchonta éstai, 211a9). Uno de los aspectos que habría que intentar aclarar es si -como supongo- las creencias previas son, al menos parcialmente, las que constituyen nuestros presupuestos comprensivos acerca del objeto de la demostración científica. Mi tesis interpretativa es que la cualificación epistémica que pertenece a tales presupuestos radica en que ellos constituyen creencias que tomamos como verdaderas acerca de qué es el objeto de la investigación -ya sea el lugar o la incontinencia-; de tal manera, los presupuestos contenidos en las creencias comunes no representan meramente un punto de partida inicial para la investigación, sino que constituyen una configuración conceptual central para llevarla a cabo, en la medida en que contienen una primera determinación acerca de la esencia de la cosa investigada (210b32). Las creencias comunes conformarían, entonces, un punto de partida necesario de toda investigación, en cuanto que configuran conceptualmente el fenómeno como objeto comprendido de cierta manera en una instancia pre-demostrativa. Para Aristóteles, las teorías científicas obtienen su punto de partida de las creencias plausibles, en cuanto que mediante ellas el científico dispone de una determinación conceptual primera e indispensable de su objeto de investigación. Ahora bien, para que una creencia esté en condiciones de ofrecer una determinación inicial del qué es debe satisfacer ciertos requisitos de verosimilitud y consistencia, los que son establecidos en una prueba dialéctica de consistencia. Sin embargo, el que ciertas creencias aprueben esa prueba no es suficiente para hacer de ellas principios explicativos de una ciencia; y en ello radica, a mi juicio, tanto la utilidad como el límite del uso dialéctico de creencias plausibles en la investigación de los principios de las disciplinas científicas.

\section{La utilización de las creencias comunes en la investigación de los principios y en la prueba de una teoría científica}

En definitiva, mi propuesta es que las dificultades interpretativas que he repasado en los apartados anteriores provienen de planteamientos que envuelven un enfoque erróneo acerca del procedimiento de una ciencia aristotélica, de su base empírica y del rol que juegan las creencias comunes en la configuración de dicha base. He propuesto -en contra de Berti- que Aristóteles no restringe la utilización de las éndoxa en el método científico a dominios de objetos donde no hay necesidad estricta, ni tampoco - en contra de Owen ${ }^{27}$ - limita su alcance a aquellos temas

27 Owen, G.E.L.: “Tithénai tà phainómena”, cit., pp. 102 s. 
donde los phainómena son creencias comunes. La consistencia, como mecanismo dialéctico de resolución de las dificultades - un procedimiento que incide en el descubrimiento de los principios (EN VII 2, 1146b7-8)-, y como índice de la verdad, aun cuando a través de ese mecanismo se alcance cierta fuerza "demostrativa", particularmente cuando el elenchus se aplica a la opinión que es contradictoria respecto de la que goza de mayor aceptación (EE I 3, 1215a6-8; Metaph. IV 4, 1006a11$18)^{28}$, creo que no debe conducirnos a una versión coherentista de la metodología de Aristóteles, en detrimento de la prioridad que ostenta la demostración estricta de los fenómenos, entendida como el procedimiento de justificación científico. Tampoco debe llevarnos a admitir una lectura apriorista de las éndoxa que las prive del contenido empírico que impregna a los "fenómenos" aristotélicos, incluso a aquellos que corresponden al dominio ético. Lo que sostengo al respecto es que el índice dialéctico de aceptación de las éndoxa -la consistencia de ciertas creencias plausibles con otras del mismo tipo fuertemente establecidas- no está disociado de la relación de acuerdo que tales creencias mantienen con nuestra experiencia y con las mejores observaciones. De esta manera, un patrón de justificación empírica no es externo a la consistencia ${ }^{29}$. En mi lectura, la condición suficiente para considerar plenamente justificado un cierto éndoxon sólo puede provenir de su integración en una teoría científica con credenciales propias, en la medida en que la demostración y su contexto científico es, para Aristóteles, el único procedimiento que puede garantizar la verdad. Es decir, se trata de creencias que deben ser justificadas al mostrarse que están de acuerdo con los fenómenos científicamente explicados, y no a la inversa. Por ello mismo, el criterio coherentista que otras interpretaciones hacen prevalecer en su explicación de la "prueba de consistencia" no puede ser aquello que el estagirita tiene a la vista cuando habla de una "prueba suficiente" (dedeigménon hikanôs, EN VII 1, 1145b7). Creo que los dos puntos centrales de mi interpretación sobre las éndoxa -es decir, (1) ellas tienen contenido empírico, y (2) realizan su aporte a la configuración conceptual de los hechos a través de una determinación

\footnotetext{
28 Berti, E.: 'L' uso 'scientifico' della dialettica in Aristotele”, cit., pp. 273 ss., esp. 280. Berti, E.: “ 'Phainomena' ed 'endoxa' in Aristotele", en W. Lapini et alii (eds.), Gli antichi e noi. Scritti in onore di Antonio Mario Battegazzore, Genova, Brigati, 2009, pp. 107-119, corrige algunos aspectos de la lectura coherentista, pero reafirma que la "demostración suficiente" consiste en que los resultados de la ciencia se vean confirmados en la medida en que ellos permiten que subsistan las éndoxa.

29 Para Bolton, el carácter plausible y común de las éndoxa depende también del hecho de que ellas son un tipo de creencias que no están en desacuerdo con los datos observacionales. Bolton, R.: "The Epistemological Basis of Aristotelian Dialectic", cit., p. 97: «This explain why Aristotle can say that in science we must try to account for the endoxa but also suppose that perceptual observations take precedence over endoxa in validating theories». Berrón, M.: Ciencia y dialéctica en Acerca del cielo de Aristóteles (inédito) (Tesis doctoral, Universidad Nacional de Rosario, Argentina, 2012), apartados 14 y 16, sostiene que la coherencia es una condición necesaria y un signo de la verdad de una tesis, pero no una condición suficiente.
} 
inicial del qué es en la instancia pre-demostrativa de una ciencia- conducen a revisar un importante conjunto de tesis de la metodología y la epistemología de Aristóteles.

La prueba dialéctica que establece como verdadera una tesis por recurso a la refutación de su contradictoria ( $E E$ I 3, 1215a5-8), a la que antes hice referencia, alcanza un estatus epistemológico necesario, y con ello parece equipararse al correspondiente rasgo formal de la epistéme (e.g. APo. I 2, 71b12, b15-16); sin embargo, en la medida en que no ofrece la causa -la segunda condición de la epistéme, 71b10, b11-, no puede satisfacer la condición de una genuina demostración aristotélica. La "demostración" por refutación no puede equipararse en manera alguna a una demostración estricta, en la medida en que ésta requiere analizar un hecho, identificarlo mediante una definición real y dar de él una explicación causal en el preciso marco formal de una apódeixis. La prueba que, por medio de la refutación de la contradictoria, podemos alcanzar de una cierta tesis se integra perfectamente, sin embargo, en la instancia pre-demostrativa de una ciencia aristotélica, es decir, en la instancia en que se trata de descubrir candidatos a principios explicativos, allí donde trato de mostrar que tiene su principal incidencia positiva el uso científico de la dialéctica.

Hay abundantes pasajes en el corpus que certifican que, en contextos científicos, el estagirita está comprometido con lo que podría denominarse el "primado epistemológico de los fenómenos de la experiencia", o de los hechos ante las creencias, incluso ante las creencias mayoritariamente aceptadas ${ }^{30}$, y no es excepción a ello el caso de la acción humana. Consideremos dos de esos pasajes; el primero, referido a la ética:

Mas acerca de todas estas cuestiones hay que buscar la certeza ${ }^{31}$ por medio de los razonamientos al usar los fenómenos como testimonios y ejemplos (martyríois kai paradeígmasi chrómenon toîs phainoménois). Lo óptimo es, pues, que todos los hombres lleguen a estar de acuerdo (phaínesthai synomologoûntas) con lo que diremos; y si no, que todos $32<$ estén de acuerdo $>$ al menos de alguna manera 33 , lo cual precisamente harán al modificar $<$ lo incorrecto $>34$. Pues cada uno posee algo propio en relación con la verdad,

30 Esto no se pone en tela de juicio por el hecho de que Aristóteles reconozca las limitaciones humanas en el conocimiento alcanzado por la observación (e.g. Cael. II 12, 292b14-17; HA I 6, 491a7-14). 31 Sobre la pistis que se obtiene demostrativamente y sobre la que corresponde a los principios, véase $A P o$. I 2, 72a25 ss. Aristóteles incluye también en la ética la investigación de la causa (EE I 6, 1216b39).

32 Leo pántas (según OCT) en EE I 6, $1216 \mathrm{~b} 30$.

33 Aristóteles supone, en este caso, que el consensus omnium es una especie de segunda mejor opción - contando como primera que haya acuerdo de las opiniones aceptadas con la teoría que se considere verdadera-, en virtud de que regularmente contiene algo verdadero. Sin embargo, incluso estas opiniones compartidas no son inamovibles, como lo indica la siguiente cláusula.

34 Para metabibázein, asociado al uso de la dialéctica, véase Top. I 2, 101a33; Metaph. VII 3, 1029b12. 
y a partir de esas $<$ opiniones $>$ es necesario probar de alguna manera aquellas $<$ cuestiones $>$. En efecto, a partir de lo dicho con verdad mas no claramente, en la medida en que avanzamos, surgirá lo <dicho $>$ claramente al sustituir ${ }^{35}$ siempre las cosas mejor conocidas $^{36}$ en lugar de 37 las habituales que se dicen confusamente 38 (EE I 6, 1216b26-35).

Y el segundo, a la ciencia natural:

Pero resulta que al hablar acerca de los fenómenos dicen cosas que no concuerdan con los fenómenos. Y de esto es causa el no tomar correctamente los primeros principios, sino querer reconducir todo a ciertas opiniones determinadas. [...] Quienes por amistad a estas <opiniones $><$ proceden así> parecen hacer lo mismo que quienes defienden celosamente las tesis en las argumentaciones. Ellos admiten, pues, cualquier conclusión, cual poseyendo principios verdaderos, como si en efecto no hubiera que juzgar algunos $<$ principios $>$ a partir de los resultados, y sobre todo a partir de su fin. [...] y el $<$ fin $>$ de la $<$ ciencia $>$ física $<$ es $>$ lo que aparece (tò phainómenon) siempre principalmente a la percepción sensible (Cael. III 7, 306a5-17).

La reivindicación aristotélica que se encuentra en estos pasajes consiste en mantener lo que -en la jerga contemporánea de John McDowell- se denomina la responsabilidad de nuestras creencias con respecto al mundo; es decir, que el parámetro de justificación de una creencia se encuentra en que ella expresa cómo son las cosas. Para Aristóteles, la plausibilidad de las éndoxa entraña su acuerdo con las mejores observaciones. Pero, en virtud de que también para Aristóteles los fenómenos de la percepción están integrados en un marco de pre-comprensión conformado por nuestras creencias comunes, el primado de tales fenómenos en el orden del descubrimiento de los principios y de la justificación de una teoría no conlleva que las observaciones estén disociadas de los conceptos que se expresan en las éndoxa y las legómena mayoritariamente aceptadas. El empirismo aristotélico no corresponde a una posición "ingenua" que le atribuye al estagirita la admisión de datos observacionales puros y duros como puntos de partida de la inducción ${ }^{39}$, sino que se debería caracterizar como un empirismo híbrido. En efecto, la empeiría aristoté-

\footnotetext{
35 Para metalambánein, véanse las referencias en Barnes, J.: "Aristotle and the Method of Ethics", cit., p. 507, n. 36 . Se trata de un paralelo con $E E$ I $6,1216 \mathrm{~b} 30$.

36 Véase Metaph. VII 3, 1029b3. El sentido corresponde a "lo que es más/mejor conocido por naturaleza" (têi phýsei).

37 Con Richards, suplemento antì ante eiothóton (EE I 6, 1216b34).

38 Para sygkechyménon, véase $P h$. I 1, 184a22. El proceso epistémico de EE I 6 guarda una estrecha relación con Ph. I 1, 184a16-18 (véanse también EE I 7, 1217a18-20; II 1, 1220a15-18), más que con $E N$ I 8, 1098b9-12, ya que el texto se centra en cómo avanzamos cognoscitivamente: cambiando lo incorrecto y reemplazando lo confuso, antes bien que apoyándonos ciegamente en los argumentos.

39 Irwin, T. H.: Aristotle's First Principles, cit., pp. 30 ss.; Owen, G.E.L.: "Tithénai tà phainómena", cit., p. 84 (PA I 1, 639b5-10, 640a13-15, citados por Owen, no son relevantes).
} 
lica, que constituye el punto de partida para la investigación de los principios, incorpora una estructuración conceptual interna provista por las éndoxa. Eso explica una propuesta metodológica de alcance programático, como la que se lee en este breve pasaje:

Pero parece que el razonamiento (lógos) testimonia (martyreîn) a favor de los fenómenos (phainoménois) ${ }^{40}$ y los fenómenos, a favor del razonamiento (Cael. I 3, 270b4-5).

Otros importantes pasajes confirman esta misma tesis. GA I 21, 729b9, destaca la concordancia entre el análisis conceptual y la evidencia empírica en la explicación del aporte del macho a la reproducción; $G A$ III 10, 760b30-33, insiste sobre la prioridad de los fenómenos observacionales ante los razonamientos; y en el texto citado de Cael. III 7 (306a5-7, 306a11-13), se critica un abordaje dogmático para descubrir los principios, lo que tiene como consecuencia que no es posible explicar los fenómenos (306a13-17). EE I 6, 1216b35 ss. advierte que proceder científicamente (philosóphos, 1216b36) requiere tanto poner de manifiesto el qué es y el por qué -conforme a la prescripción de APo. II 1-como también ofrecer razonamientos sobre la base de principios que pertenezcan al mismo género que lo demostrado -en conformidad con APo. I 7; I 9, 76a27-30-. En Cael. II 13, 293a25-27, el trasfondo metodológico de la objeción a la teoría pitagórica de la anti-Tierra descansa en que los testimonios observacionales gozan de una autoridad independiente y no se legitiman por su relación de concordancia con los argumentos y las creencias. Todos estos documentos sugieren que un argumento y los fenómenos a los cuales aquél se refiere no pueden alegarse por separado como justificación.

Así, el sentido que adquiere justificar el resultado de una investigación por medio de su consistencia con las creencias comunes, que se encuentra formulado en $E N$ VII 1 y $P h$. IV 4, no quiere decir que la "demostración suficiente" postulada en esos pasajes pueda hacerse por mero recurso a la coherencia, ni que las creencias comunes utilizadas como premisas de la prueba dialéctica se hallen desligadas de los hechos de la experiencia y de su posible explicación científica. Habiendo remo-

\footnotetext{
40 Moraux, P.: Aristote, Du Ciel. Texte établi et traduit, Paris, Les Belles Lettres, 1965, p. 8, traduce por "opinion commune". Sin embargo, Aristóteles incluye en estas consideraciones tanto cosas dichas (e.g. Cael. I 2, 268b15) como afirmaciones basadas en observaciones (e.g. 269a2 ss.); y ambos factores componen las premisas de los razonamientos que efectúa (269b13-17). Expresiones como eúlogon hypolabeîn (I 3, 270a12) o tês autês dianoías estìn hypolabeîn (270a26) y toîs hypokeiménois pisteúei (270b3) no sugieren un método lógico ya que en las premisas se incorporan aquellos dos componentes. Que en el pasaje traducido se trata de fenómenos observacionales lo confirma 270b11-12: «Pero esto resulta también suficientemente a través de la percepción sensible (aisthéseos)», donde "esto" remite a la concepción (hypólepsis, 270b6) universalmente extendida acerca de lo divino, con la cual coinciden las observaciones sensibles. Véase Jori, A.: Aristoteles, Über den Himmel. Übersetzt und erläutert, Berlin, Akademie, 2009, p. 386.
} 
vido algunos problemas aparentes, y enfocado desde la óptica de un empirismo híbrido la doble constitución de la empeiría aristotélica a través de datos observacionales y conceptos endóxicos, creo que estamos ahora en una mejor posición para determinar dónde pueden efectuar las éndoxa su aporte positivo a la investigación de los principios.

\section{El rol de las creencias comunes en la ciencia natural: éndoxa y definición}

La aplicación y la utilidad científica de las creencias plausibles puede entenderse si consideramos un pasaje que fuera leído incorrectamente en clave coherentista por Owen. En efecto, la ubicación del uso científico de las éndoxa en la instancia pre-demostrativa nos permite entender por qué el libro primero de la $P h$. se aboca a consideraciones lingüísticas acerca de la manera en que hablamos, o sea, examina legómena referidas al cambio ( $P h$. I 7). Para ello, Aristóteles recurre a una herramienta claramente identificada ya en la exposición de los instrumentos de que se sirve la dialéctica, en Top. I 15: la distinción de significados es una herramienta que apela a la distinción categorial -Top. I 9- y a aspectos centrales de la semántica aristotélica, como la teoría de los términos contrarios, la teoría de la definición por género y diferencia específica, así como también a otras teorías de mayor -la teoría de los predicables- y menor envergadura - e.g. la referida a la inflexión gramatical de los nombres, que se utiliza como índice para el análisis de la polisemia-. Enfoquemos ahora uno de los textos más importantes para la metodología aristotélica que me propongo considerar en la parte final de este trabajo. En Ph. IV 4, 210b32-211a11, Aristóteles escribe:

Qué es (tí estín, 210b32) el lugar llegaría a ser manifiesto de la siguiente manera. Tomemos (lábomen, 210b32-33) acerca de él cuantas cosas parecen verdaderamente corresponderle por sí mismo (hósa dokeî alethôs kath'hautò hypárchein autôi, 210b3334). Sostenemos básicamente (axioûmen, 210b34) ahora que el lugar es lo primero que contiene aquello de lo cual <él> es lugar, y que no es nada del objeto [...] ${ }^{41}$. Y bien, suponiendo (hypokeiménon, 211a6) tales cosas hay que considerar (theoretéon, 211a67) las restantes. Pero es necesario intentar realizar la investigación (sképsin) de tal modo que será dado el qué es (tò tì ésti, 211a8), de manera que se resuelvan las aporías (tà aporoúmena, 211a8) y las opiniones (tà dokoûnta, 211a9) que corresponden al lugar serán las que corresponden, y además se hará manifiesta la causa de la dificultad y de las aporías acerca de aquel. Pues así cada cosa se probaría de la mejor manera (kállista deiknýoito, 211a11).

41 Ésta es la primera de seis propiedades por sí del tópos (Ph. IV 4, 210b34-211a6). 
Aristóteles plantea aquí un procedimiento para conocer la esencia (tò tí esti) de un objeto cuya investigación comenzó en el libro precedente en directa relación con la definición del movimiento. Sabemos que para Aristóteles no hay demostración de la esencia (APo. II 4; I 3, 72b18-32; Top. I 2, 101a37-b4; Metaph. VI 1, 1025b1018; Ph. II 1, 193a1-9)42, y que un recurso para establecerla es la división ${ }^{43}$. Aristóteles no está aplicando aquí ese procedimiento, pero emplea otro dialéctico, el examen de las éndoxa, cuya meta coincide parcialmente con la división, en cuanto que también por intermedio de aquél se busca determinar las propiedades que pertenecen por sí al objeto.

$P h$. IV 4 ejemplifica la situación epistemológica de $A P o$. I 1, donde se mantiene que toda elaboración de conocimiento parte de conocimientos preexistentes (71a1-2). En el caso de la esencia, su conocimiento debe alcanzarse de manera inductiva (71a5-6). Según Metaph. VI 1, 1025b10-12 y APo. II 3, la esencia se conoce a través de los sentidos o se presupone como una hipótesis. El examen dialéctico de las éndoxa realiza un aporte de fundamental importancia en este último aspecto, ya que las opiniones acreditadas constituyen aquello que suponemos acerca de lo que es cierto objeto. Las éndoxa son, entonces, un componente principal de la base inductiva para la generalización ( $A P o$. I 1, 71a8-9) que se lleva a cabo al suponer una definición tanto del objeto como de sus atributos por sí. En $P h$. IV 4, 210b33-34, se parte de las éndoxa que transmiten una información acreditada acerca de los atributos que le pertenecen por sí al lugar, esto es, que se hallan vinculados a qué es el lugar (211a8); y a partir de tal hipótesis se alcanza una definición inicial (211a7-8), que, ciertamente, no es la definitiva ya que ésta podrá establecerse sólo en el curso de la demostración 44 .

La selección de las premisas (tàs protáseis eklektéon, Top. I 14, 105a34) es una de las herramientas dialécticas individuadas en Top. I 13; la misma que aplica $P h$. IV 445. En ella se trata de examinar ciertas opiniones aceptadas (I 14, 105a35-37; 1,

42 Berti, E.: "Les méthodes d'argumentation et de demonstration dans la 'Physique'. (Apories, Phénomènes, principes)", en F. De Gandt et P. Souffrin (eds.), La Physique d'Aristote et les conditions d'une science de la nature, Paris, Vrin, 1991, pp. 53-72, pp. 68-70, pp. 57, 66 s.; Bolton, R.: "Aristotle's Method in Natural Science: Physics I", cit., pp. 2-13.

43 Para su empleo, APo. II 13.

44 Bolton, R.: "Aristotle's Method in Natural Science: Physics I", cit., pp. 19 ss. También sobre "grave" y "leve" se formulan definiciones con un estatuto preliminar semejante (Cael. I 3, 269b20-23).

45 Los cuatro órgana para descubrir los elementos (Top. I 13, 105a22) de un razonamiento dialéctico son: (1) tomar las premisas (I 14); (2) distinguir los sentidos de los términos (I 15); (3) hallar las diferencias (I 16); y (4) observar lo semejante (I 17). Ellos indican procedimientos para formular una premisa (poiêsai prótasin, I 13, 105a27) de un silogismo (sobre syllogismós y lógos, I 1, 100a25-27) y son complementarios respecto de los géneros acerca de los cuales, y respecto de los elementos a partir de los cuales (tà mèn oûn géne perì hôn te hoi lógoi kaì ex hôn, I 13, 105a20) se formulan los enunciados (lógoi) que se integran en razonamientos (105a20-21); es decir, aquello sobre lo cual y a partir de lo cual se formula un enunciado, eso delimita su contenido proposicional, tal como éste se consi- 
100b21-23; 10, 104a8-10). Es importante para mi argumento subrayar el peso epistémico que adquiere esta herramienta, y mostrar, al menos a través de pasajes representativos, que ese instrumento se aplica de manera sistemática en la investigación de los principios. En Top. I 14, Aristóteles refuerza el carácter epistémico del cual gozan las creencias resultantes del proceso de selección, indicando que las opiniones elegibles no deben ser contrarias a los fenómenos (tàs $<m e ̀>$ enantías taîs phainoménais, I 14, 105a37-b1). Él evita, así, que entre las opiniones seleccionadas para ser sometidas al examen dialéctico se incluyan opiniones exóticas de los expertos, las cuales no están de acuerdo con las mejores observaciones y carecen de fuerza endóxica; similarmente, excluye las opiniones sólo aparentemente endóxicas (tò phainómenon éndoxon, I 1, 100b26). Una prescripción metódica del estagirita, que encontramos en GA III 10, 760b27-33 y se halla estrechamente relacionada con esto último, indica rechazar opiniones reputadas que no fueron establecidas a partir de una descripción empíricamente adecuada de los hechos relevantes. Tales opiniones podrían catalogarse como sólo aparentemente endóxicas. Esto permite entender que Aristóteles tenga en cuenta opiniones plausibles para rechazar ciertas opiniones de expertos, en la medida en que estas últimas resulten exóticas o contrarias a los fenómenos, sean éstos observacionales (phainómena katà tèn aísthesin) (Cael. III 4, 303 a20-23 $)^{46}$ o pertenezcan a la clase de las opiniones acreditadas. En este último caso, se recusan las opiniones de los expertos alegando que contradicen otras creencias que exhiben buenas credenciales para su sostenimiento y aceptación (e.g. Ph. I 8, 191b30-31). Un último aspecto importante sobre la selección de las premisas es que de aquellas opiniones de cuya plausibilidad es índice la aceptación mayoritaria, son elegibles particularmente cuantas opiniones guardan conformidad con las ciencias (hósai dóxai katà téchnas, Top. I 14, 105b1). En este caso, la procedencia del índice de plausibilidad 47 reside en las virtudes epistémicas del conocimiento científico. El examen dialéctico que se aplica en nuestro pasaje de $P h$. IV 4 respeta estos

dera dentro de la prescripción dialéctica de Top. (ek tínon he méthodos, I 4, 101b11; pròs pósa kaì poîa kaì ek tínon hoi lógoi, 101b12-12; perì hôn, 101b16; apò toúton, 101b27-28). Una premisa o proposición, al igual que un problema -lo que aborda un razonamiento dialéctico (I 2, 101b28-36; el paralelo entre prótasis y próblema, en I 14, 105b25)-, expresa algunos de los cuatro predicables (I 4, 101b17-18; I 5). Aristóteles se propone, por ende, mostrar para cada uno de los lugares de enunciación o predicables de qué manera pueden ser útiles las herramientas argumentativas antes mencionadas (I $18,108 \mathrm{~b} 32-33)$. Entre lugares y herramientas hay una complementariedad que atañe, entonces, a la relación que existe entre premisa y argumento.

46 Véanse especialmente dos pasajes fundamentales: Cael. III 7, 306a5-17 y GC I 2, 316a5-14; así como también Cael. II 13, 293a23-27, 294a ss.; III 4, 303a22-23; GA I 18, 725b5-6; GC I 1, 315a3-4; Metaph. XII 8, 1073b36-1074a5. Cleary, J. J.: Aristotle and Mathematics, cit., pp. 134 ss., insiste en que, para Aristóteles, las hipótesis con que opera metódicamente el científico natural deben someterse al tribunal de los fenómenos.

47 En Top. I 14, se alude varias veces $(105 \mathrm{~b} 2,105 \mathrm{~b} 4,105 \mathrm{~b} 18,105 \mathrm{~b} 10-11)$ al parámetro de la plausibilidad como aquel que deben cumplir las premisas seleccionadas; y, por lo tanto, tal es un elemento de prueba en la utilización de esa herramienta. 
parámetros metodológicos. En 210b34-211a6, Aristóteles enumera seis principales axiómata acerca del lugar, que tienen el rango de opiniones plausibles obtenidas a partir de la discusión dialéctica precedente (IV 1-3), y se introducen con el objetivo de averiguar la esencia del lugar. ${ }^{48}$

\section{El descubrimiento dialéctico de los principios y su lugar en la epistéme}

El uso de las éndoxa en $P h$. IV 4 forma parte del método que plantea recurrir a la empeiría para descubrir principios, formulado programáticamente en $A P r$. I 30, 46a18-19. Esto no contradice que en la ciencia natural la percepción sea la fuente principal para la inducción de principios. Pues, en la concepción de Aristóteles, la percepción es una fuente epistemológica compleja, cuya articulación comprensiva está dada por las nociones y los términos lingüísticos que provienen, precisamente, de las creencias admitidas. De allí que el examen dialéctico de las éndoxa forme parte del mismo método de análisis de los conceptos y de nombres como "círculo", cuyo estatuto epistemológico se califica en $P h$. I 1, 184a23-184b14 como el correspondiente a una generalidad indiferenciada (hólon [...] adioristos, 184b2) y a algo complejo (sygkechyménon, 184a22). Esto sirve para caracterizar desde un punto de vista formal-epistemológico los correlatos de la percepción, en cuanto que ellos son cosas más conocidas para nosotros (184a21-22; en general, 184a16 ss.). Hacer evidente mediante un argumento inductivo basado en la percepción (APo. II 19, 100b3$5)$ cierto factor que constituye un candidato a principio - e.g. el movimiento de las entidades naturales, para la física ( $P h$. I 2, 185a13-14)-, es algo que debe entenderse, por consiguiente, como un procedimiento empírico-inductivo híbrido, que compone la empeiría a través de una combinación entre percepción, creencias comunes y nombres o descripciones lingüísticas. Resulta, entonces, perfectamente coherente con esta metodología de análisis que la definición -en cuanto que ella contiene cosas más conocidas por sí, i.e. los componentes o partes de la esencia-se halle con el nombre -lo más conocido para nosotros- en una relación explicativa semejante $(184 \mathrm{a} 26-\mathrm{b} 12)^{49}$ :

[...] pues $<$ el nombre $>$ significa cierta totalidad e indeterminadamente, por ejemplo, el "círculo", mientras que su definición $<\mathrm{la}>$ divide en los $<$ componentes $>$ particulares (184b11-12).

\footnotetext{
48 Vigo, A.: Aristóteles, Física: Libros III-IV. Traducción, introducción y comentario, Buenos Aires, Biblos, 1995, p. 191.

49 Wagner, H.: Aristoteles, Physikvorlesung. Übersetzt, Berlin, Akademie, 19955, pp. 394 s.
} 
En algunos trabajos recientes se acentúa el aspecto disposicional de la empeiría y la epagogé aristotélica, en términos de la descripción de Metaph. I 1, 980b28 ss. ${ }^{50}$. Sin embargo, un pasaje como GC I 2, 316a5-16 debería servir para poner límites a una lectura unilateralmente psicológica de la empeiría, que va en desmedro de su carácter metódico. Aristóteles critica a los atomistas por su apeiría, que los condujo a postular principios impropios y falsos. Este texto formula una impugnación metodológica a las teorías abstractas - la de las magnitudes indivisibles-, las cuales, en virtud de su insuficiencia en cuanto a la recopilación de datos relevantes (apeiría , 316a6), no son capaces de tomar en consideración las cosas sobre las cuales existe consenso (tà homologoúmena, 316a5). Quienes son alcanzados por esta crítica carecen de la habituación necesaria para abordar fenómenos naturales y no consideran suficientemente los hechos ${ }^{51}$. Aristóteles impulsa, entonces, una educación de experto en la observación de la naturaleza como condición necesaria para descubrir los principios.

Si bien la inducción aristotélica representa, antes bien que un procedimiento pautado, una capacidad mental que, mediante un acto de atención, nos permite captar algo universal como consecuencia de atender a ciertos rasgos particulares ${ }^{52}$, ella se integra en un procedimiento metódico. Su rédito principal consiste en poner de manifiesto con evidencia (dêlon) hechos generales. Pero el rasgo de la evidencia es algo que puede obtenerse tanto a partir de la percepción (en el caso del movimiento) como también a partir del examen dialéctico de las opiniones de los expertos (la opinión -que se convertirá en uno de los principios del movimiento y de la ciencia natural, Ph. I 7, $190 \mathrm{~b} 35$ ss.- que explica la kínesis como una transición entre contrarios, I 5, 188a26-27).

\section{Dialéctica y ciencia en $P h$. IV 4}

Dedicaré el último apartado de este trabajo a mostrar brevemente en el texto de $P h$. IV 4 la incidencia de los elementos metodológicos que hasta aquí he tratado de

\footnotetext{
50 Con el antecedente de Mansion 19872, 217 ss.; no obstante, él (p. 223, n. 36) reconoce el componente netamente empírico de la epagogé. La inducción en el dominio de la ciencia natural equivaldría a la constatación observacional de hechos generales repetidos del mundo físico que ostentan algún patrón de semejanza y se hallan ya interpretados. La existencia del movimiento, que es parte de la noción de naturaleza (II 1, 192b13-14) y un principio de esta ciencia, se conoce también "a partir de la inducción" (I 2, 185a13-14).

51 Joachim, H. H.: Aristotle, On Coming To Be \& Passing-Away. A Revised Text with Introduction and Commentary, Oxford, Oxford University Press, 1926, ad 316a8-10.

52 Engberg-Pedersen, T.: "More on Aristotelian Epagoge", Phronesis, XXIV, 1979, pp. 301-30; la lectura ortodoxa en Ross, W. D.: Aristotle's Prior and Posterior Analytics. A Revised Text with Introduction and Commentary, Oxford, Oxford University Press, 1949, pp. 47 ss.
} 
destacar. El segundo tema del texto de IV 4 (211a7-11) se refiere al método de investigación que parte de las opiniones comunes e intenta obtener una primera determinación de qué es el lugar (211a8). Una vez establecida su definición en términos del límite primero inmóvil del cuerpo continente (212a20-21; 5, 212a31-32, que retoma lo dicho previamente en 4, 211a31-33), las dificultades concernientes al conocimiento de la esencia y de los atributos por sí del lugar podrán resolverse. Conjuntamente, las creencias que fueron consideradas como conducentes para el fin anterior resultarán finalmente establecidas como genuinas descripciones de los hechos, es decir, como verdaderas y, además, explicadas ya que los factores del hecho que ellas introducen (e.g. la distinción entre objeto y lugar, introducida en las éndoxa segunda y cuarta; 4, 211a1-3) se demuestran.

Ph. IV 4 muestra ejemplarmente la utilidad científica de la dialéctica. Aristóteles distingue la relación que mantiene un cuerpo físico con el lugar que ocupa respecto de la que mantiene la mano con el cuerpo al que está ligada; para hacerlo, apela a nociones que delimitan el dominio específico de la región de esta ciencia ("continente", "continuidad", "contacto", "estar dividido", "ser parte de", "ser un todo", etc., $211 \mathrm{a} 29$ ss.), y que permiten hacer conocido qué es el lugar (211b5-6). Ahora bien, esos conceptos-marco están respaldados por su aspiración epistemológica de captar los fenómenos relevantes (e.g. un cuerpo que se halla separado o dividido de otro, o sea, que no es continuo con otro, pero que, sin embargo, está en contacto con él, no se desplaza con ese cuerpo, sino en él; 211a35-36). Esta última consideración (211a36-b1) tiene el valor de anticipar la solución a un posible problema que podría plantearse a la distinción entre, por un lado, la relación entre un líquido y el recipiente que lo contiene, y, por otro, la que existe entre una parte y el todo (211b1-5). En efecto, el movimiento del cuerpo que está en un lugar no depende de que el lugar se mueva, como en el caso de lo que es una parte de un todo mayor. El rango epistémico de estas consideraciones consiste en profundizar distinciones conceptuales, mostrando que un aspecto del fenómeno -la relación parte/todo- no constituye una prueba en contrario de la propuesta previamente efectuada, ya que no se verifica en los casos que se entienden como la ocupación de un lugar.

Como fuera señalado53, en $P h$. IV 4 asistimos a un examen dialéctico de las éndoxa. Sin embargo, he tratado de mostrar que el recurso a distintas herramientas dialécticas, en éste y otros tratados de ciencia natural, no implica que las éndoxa carezcan de base empírica ni que su utilización sea infructuosa en la formulación de nociones básicas y específicas. Por lo tanto, hay que concluir que la utilización de éndoxa forma parte de la aplicación de un método empírico. Algunos de los pasajes representativos que he considerado hasta aquí permiten confirmar que el objetivo

53 Owen, G. E. L.: “Tithénai tà phainómena”, cit., pp. 92 ss., «[...] the work is logical [...]» (p. 103). 
de la ciencia natural, consistente en construir explicaciones de sus propios fenómenos, impone al uso científico de la dialéctica un contexto de interés prioritario -el de la explicación causal- que relega el parámetro dialéctico de la consistencia y convierte a la herramienta dialéctica de la selección de las premisas en un componente de la investigación científica que incluye aquellas creencias cuyo contenido expresa algo verdadero acerca de los hechos. En la medida en que fenómenos y creencias conforman los dos componentes diferenciados y complementarios, pero con prioridad del primero, de la base inductiva de una ciencia aristotélica, ambos constituyen el empirismo híbrido que es la marca de fábrica de la metodología de Aristóteles.

\section{Referencias bibliográficas}

BARnes, J.: "Aristotle and the Method of Ethics", Revue International de Philosophie, 34, 1980, pp. 490-511.

Berrón, M.: Ciencia y dialéctica en Acerca del cielo de Aristóteles (inédito) (Tesis doctoral, Universidad Nacional de Rosario, Argentina, 2012).

BERTI, E.: "Les méthodes d'argumentation et de demonstration dans la 'Physique'. (Apories, Phénomènes, principes)", en F. De Gandt et P. Souffrin (eds.), La Physique d'Aristote et les conditions d'une science de la nature, Paris, Vrin, 1991, pp. 53-72.

BerTi, E.: 'L' uso 'scientifico' della dialettica in Aristotele", reed. en Nuovi studi aristotelici. I- Epistemologia, logica e dialettica, Brescia, 2004, Morcelliana, pp. 265-282 (cit. según ed. 1995).

BERTI, E.: "Il valore epistemologico degli éndoxa secondo Aristotele", reed. en Nuovi studi aristotelici. I- Epistemologia, logica e dialettica, Brescia, 2004, Morcelliana, pp. 317-332 (cit. según ed. 2001-2002).

BerTI, E. “'Phainomena' ed 'endoxa' in Aristotele”, en W. Lapini, W., Malusa, L., Mauro, L. (eds.), Gli antichi e noi. Scritti in onore di Antonio Mario Battegazzore, Genova, Brigati, 2009, pp. 107-119.

Bolton, R.: "Aristotle's Method in Natural Science: Physics I", en Judson, L. (ed.), Aristotle's Physics. A Collection of Essays, Oxford, Oxford University Press, 1991, pp. 1-29.

Bolton, R.: "The Epistemological Basis of Aristotelian Dialectic", en Sim, M. (ed.), From Puzzles to Principles? Essays on Aristotle's Dialectic, Lanham, Lexington, 1999, pp. 57-105.

Bolton, R.: "Two Standards for Inquiry in Aristotle's De Caelo", en Bowen, C. A. and Wildberg, C. (eds.), New Perspectives on Aristotle's De Caelo, Leiden/Boston, Brill, 2009, pp. 51-82. 
Brunschwig, J.: Aristote, Topiques (Livres I-IV). Texte établi et traduit, Paris, Les Belles Lettres, 1967.

Charlton, W.: Aristotle, Physics. Books I and II. Translated with Introduction, Commentary, Note on Recent Work, and Revised Bibliography, Oxford, Oxford University Press, 1992.

Cleary, J. J.: Aristotle and Mathematics. Aporetic Method in Cosmology and Metaphysics, Leiden, Brill, 1995.

Engberg-Pedersen, T.: "More on Aristotelian Epagoge", Phronesis, XXIV, 1979, pp. 301-309.

InwIN, T. H.: Aristotle's First Principles, Oxford, Oxford University Press, 1988.

JoAchim, H. H.: Aristotle, On Coming To Be \& Passing-Away. A Revised Text with Introduction and Commentary, Oxford, Oxford University Press, 1926.

JoRI, A.: Aristoteles, Über den Himmel. Übersetzt und erläutert, Berlin, Akademie, 2009.

MANSION, A.: Introduction à la physique aristotélicienne, Louvain-La-Neuve, Éditions de 1'Institut Supérieur de Philosophie, 19872.

Moraux, P.: Aristote, Du Ciel. Texte établi et traduit, Paris, Les Belles Lettres, 1965.

Owen, G. E. L.: “Tithénai tà phainómena", en S. Mansion (ed.), Aristote et les Problèmes de Méthode, Paris/Louvain, Éditions de l'Institut Supérieur de Philosophie, 19802, pp. 83-103.

Ross, W. D.: Aristotle's Prior and Posterior Analytics. A Revised Text with Introduction and Commentary, Oxford, Oxford University Press, 1949.

Smith, R.: "Dialectic an Method in Aristotle", en M. Sim (ed.), From Puzzles to Principles? Essays on Aristotle's Dialectic, Lanham, Lexington, 1999, pp. 3955.

Vigo, A.: Aristóteles, Física: Libros III-IV. Traducción, introducción y comentario, Buenos Aires, Biblos, 1995.

Wagner, H.: Aristoteles, Physikvorlesung. Übersetzt, Berlin, Akademie, 19955.

WiEland, W.: Die aristotelische Physik. Untersuchungen über die Grundlegung der Naturwissenschaft und die sprachlichen Bedingungen der Prinzipienforschung bei Aristoteles, Göttingen, Vandenhoeck \& Ruprecht, $1992^{3}$.

ZAdro, A.: Aristotele, I Topici. Traduzione, introduzione e commento, Napoli, Loffredo, 1974.

Fabián Mié

Consejo Nacional de Investigaciones Científicas y Técnicas (CONICET)

Universidad Nacional del Litoral

fabian.mie@conicet.gov.ar 